\title{
LATERAL PRESSURES ON RETAINING WALLS DUE TO LOADS ON SURFACE OF GRANULAR BACKFILL
}

\author{
Banabihari Misra*
}

\section{ABSTRACT}

The lateral pressure transmittal through different types of backfill material; characterized through a material parameter $E / G$; subjected to different types of contact pressure on its surface is evaluated theoretically by the use of the concept, in which it is considered that $E / G>2(1+\nu)$. The backfill material extending upto infinity depth as well as limited by the presence of rigid boundaries having different frictional characteristics have been considered. The physical and mechanical characteristics of the granular materials affecting the development of lateral pressures on retaining wall are investigated. The model used in the analysis is found to be applicable to a wide range of materials ranging from solid like at one end to liquid like at the other end.

Qualitative numerical results over a wide range of the values of the material parameter $(E / G)$ have been presented in a non-dimensional form to elucidate its influence on this problem. The results are also compared with that of Boussinesq's approach. It is observed that the softer/looser the backfill, the poorer is pressure transmission in horizontal direction resulting in a overall decrease of the lateral pressure on the retaining structure. The proposed model gives a partial explanation of the divergences between the results of the existing theories and reality.

Key words: backfill, decomposed granite soil, earth pressure, elasticity, granular material, retaining wall, soft ground, stress, uniform load

IGC: $\quad \overline{\mathrm{E} 5 / \mathrm{H} 2}$

\section{INTRODUCTION}

Lateral pressures due to surcharge loads superimposed on the surface of a backfill have received relatively little attention from researchers, especially those based on analytical works involving theory of elasticity (Spangler, 1938; Spangler and Mickle, 1956; Richard and Linger, 1965). Designers are forced to rely, to a much greater extent, upon rule-of-thumb procedures and individual judgement and intuition(Hansen, 1953; Tschebotarioff, 1957; Terzaghi, 1943). This method of approach has several short comings and disadvantages.

This rule utilizes an additional depth of backfill in the calculations of lateral pressure due to surcharge loads; resulting a uniform intensity throughout the entire height of the wall, regardless of the position or the shape and degree of concentration of the actual surface load. Research in recent years has indicated rather definitely

* Assistant Professor, Department of Civil Engineering, Indian Institute of Technology, Kharagpur, India.

Written discussions on this paper should be submitted before April 1, 1981. 
that this pattern of pressure distribution does not coincide with fact (Barber, 1956; Richard and Linger, 1965). Their findings, as well as the experimental evidence of Spangler and Mickle (1956), showed that the intensity of lateral pressure due to surface load is maximum near the surface and diminishes rapidly in intensity with depth.

\section{APPLICATION OF ELASTIC THEORY}

The investigations involving analytical works are mostly based on Boussinesq's (1885) classical elastic theory. Boussinesq's stress distribution theory was for the simplest case of loading of a solid which was considered to be a homogeneous, elastic, isotropic, semi-infinite medium. Boussinesq's equation for radial stress, expressed in cylindrical polar co-ordinates, due to a concentrated load $(P)$ on the ground surface is given by (Timoshenko, 1934);

$$
\sigma_{r r}=\frac{P}{2 \pi}\left[3 r^{2} z\left(r^{2}+z^{2}\right)^{-5 / 2}-(1-2 \nu)\left[r^{-2}-z r^{-2}\left(r^{2}+z^{2}\right)\right]^{-1 / 2}\right]
$$

in which, $r$ and $z$ are respectively the radial and depth co-ordinates, and $\nu$ is the Poisson's ratio, the range of which varies from $\nu=0$ to $\nu=0.5$.

Because of the fact that Poisson's ratio for soils has always been very difficult to ascertain; for simplicity researchers (Spangler and Mickle, 1956; Richard and Linger, 1965) have worked with $\nu=0.5$, irrespective of the nature of the backfil material. Thus Eq. (1) simplifies to,

$$
\sigma_{r r}=\frac{3 P}{2 \pi} r^{2} z\left(r^{2}+z^{2}\right)^{-5 / 2}
$$

The usefulness, limitations and short comings of Boussinesq's solutions in predicting the pattern of behaviour of granular masses subjected to surface load have been reported by several researchers (available in the reference listed under Spangler, 1938) and also by Misra and Sen $(1975,1976)$. These are equally applicable to the retaining wall problem; that is to lateral pressure transmittal due to external load on the surface of a granular backfill.

\section{Lateral Pressures Against a Vertical Retaining Wall Due to Surface Loads}

The half-space analysis deals with a medium of infinite extent in both the horizontal directions; and under an external load every point undergoes a deflection to a new position. It is assumed that a retaining wall is immovable and therefore prevents the deflection of elements adjacent to it in a direction normal to its surface. It has been shown; theoretically by the method of images (Mindlin, 1936; Weiskopf, 1945). and now widely accepted; that the pressure on a smooth rigid wall is exactly double the radial direct stress values given by the half-space solution.

\section{SCOPE OF THE INVESTIGATION}

There is very little evidence, in the literature of work being done-theoretical (Weiskopf, 1945)/experimental (Matsuo et al., 1978) - on the subjects of comparison of lateral pressure transmittal with different soils as backfill material. The problem investigated in this paper concerns the transmittal of lateral pressure through a discrete, particulate granular bakfill to a retaining structure (Massarsch and Broms, 1976; Ochiai, 1977). The aim of this theoretical investigation was to determine the variation of lateral pressure distribution resulting from different types of backfill soil under wheel loads, taking into account its material properties (Ichihara and Matsuzawa, 1970). 
Thus, based on the new information obtained (Misra and Sen, 1975-1976) from the concept of relating the stress distribution in materials to the ratio $E / G$, in which it is considered that $E / G>2(1+\nu)$; it was thought worth while to further investigate the influence of the material parameter $(E / G)$ on this type of problem. In this model the amount of horizontal load distribution is no longer fixed by the almost inflexible relation between the modulus of elasticity in compression $(E)$ and the modulus of elasticity in shear $(G)$ which is the basis of Boussinesq's approach.

This material parameter $(E / G)$ can have different values, between 2 and infinity, for different material structures, such as; solids, solid materials (firm rocks and deposites), loose particulate materials-dry or saturated to different degrees-sensitive grain structures (quick sand, sensitive clays, soft soils like marginal lands etc.) and liquid like materials (liquefaction, piping etc.). It is expected that the softer/looser the medium the higher will be the material parameter $(E / G)$ because of the poor shearing resistance of such materials.

\section{BASIC EQUATIONS FOR GRANULAR MATERIALS}

By solving the axisymmetric equations of equilibrium for granular materials with the model, $E / G>2(1+\nu)$, the writer (1975) has shown that, in cylindrical polar coordinates $(r, \theta, z)$, the stresses and dispacements can be expressed in terms of two stress functions $\phi(r, z)$ and $\phi(r, z)$; and they are as follows:

$$
\begin{aligned}
& \sigma_{r r}(r, z)=\frac{\partial^{2} \phi}{\partial z^{2}}+\frac{1}{r} \frac{\partial}{\partial r}(\psi+K \phi) \\
& \sigma_{\theta \theta}(r, z)=\nu \nabla^{2} \phi-\frac{1}{r} \frac{\partial}{\partial r}(\psi+K \phi) \\
& \sigma_{z z}(r, z)=\frac{\partial^{2} \phi}{\partial r^{2}}+\frac{1}{r} \frac{\partial \phi}{\partial r} \\
& \tau_{r z}(r, z)=-\frac{\partial^{2} \phi}{\partial r \partial z} \\
& u(r, z)=\frac{1+\nu}{E} \frac{\partial}{\partial r}(\psi+K \phi) \\
& w(r, z)=\frac{1+\nu}{E} \frac{\partial}{\partial z}(\psi-\phi)
\end{aligned}
$$

where, $\nabla^{2}$ is the Laplacian operator

$$
K=\frac{1}{1+\nu}\left[\frac{E}{G}-(1+\nu)\right]>1
$$

and $\phi(r, z), \phi(r, z)$ must satisfy the following coupled system of partial differential equations;

$$
\begin{aligned}
\nabla^{4} \phi & =\frac{1-K}{1-\nu} \frac{\partial^{2}}{\partial z^{2}}\left(\nabla^{2} \phi-\frac{\partial^{2} \phi}{\partial z^{2}}\right) \\
\nabla^{4} \psi & =\frac{1-K}{1-\nu} \frac{\partial^{2}}{\partial z^{2}}\left(\nabla^{2} \psi-\frac{\partial^{2} \psi}{\partial z^{2}}\right) \\
\frac{\partial^{2} \psi}{\partial z^{2}} & =(1-\nu) \nabla^{2} \phi
\end{aligned}
$$

The solution of the Eqs. (10) through (12) for the half-space problems are determined by the writer (1975) as;

$$
\phi(r, z)=\int_{0}^{\infty}\left(A e^{\alpha \lambda z}+B e^{-\alpha \lambda z}+C e^{\beta \lambda z}+D e^{-\beta \lambda z}\right) \lambda J_{0}(\lambda r) d \lambda
$$




$$
\psi(r, z)=\int_{0}^{\infty}\left[L\left(A e^{\alpha \lambda z}+B e^{-\alpha \lambda z}\right)+M\left(C e^{\beta \lambda z}+D e^{-\beta \lambda z}\right)\right] \lambda J_{0}(\lambda r) d \lambda
$$

where, $J_{0}$ is the Bessel's function of first kind of order zero and the effect of the material parameter $(E / G)$ is reflected in the stress and deformation equations through the two coefficients, $\alpha$ and $\beta$; where $\alpha^{2}$ and $\beta^{2}$ are the roots of the equation,

$$
x^{2}+\left(K^{\prime}-2\right) x+1=0
$$

in which, $K^{\prime}=(1-K) /(1-\nu) L=(K-1) /\left(\alpha^{2}-1\right), \quad M=-\alpha^{2} L$, and $A, B, C$ and $D$, are arithmatic functions of the trigonometric variable $(\lambda)$ to be determined consistent with the boundary conditions of the problem to be solved.

Thus, the stress and displacement Eqs. (3) through (8) yields;

$$
\begin{aligned}
\sigma_{r r} & =F_{1}(r, z)-F_{2}(r, z) / r \\
\sigma_{\theta \theta} & =F_{2}(r, z) / r+\nu\left[F_{1}(r, z)-F_{3}(r, z)\right] \\
\sigma_{z z}= & -F_{3}(r, z) \\
\tau_{r z}= & \int_{0}^{\infty}\left[\alpha\left(A e^{\alpha \lambda z}-B e^{-\alpha \lambda z}\right)+\beta\left(C e^{\beta \lambda z}-D e^{-\beta \lambda z}\right)\right] \lambda^{3} J_{1}(\lambda r) d \lambda \\
u= & \frac{1+\nu}{E} F_{2}(r, z) \\
w= & \frac{1+\nu}{E} \int_{0}^{\infty}\left[\alpha(L-1)\left(A e^{\alpha \lambda z}-B e^{-\alpha \lambda z}\right)\right. \\
& \left.+\beta(M-1)\left(C e^{\beta \lambda z}-D e^{-\beta \lambda z}\right)\right] \lambda^{2} J_{0}(\lambda r) d \lambda
\end{aligned}
$$

where,

$$
\begin{aligned}
& F_{1}(r, z)= \int_{0}^{\infty}\left[\alpha^{2}\left(A e^{\alpha \lambda z}+B e^{-\alpha \lambda z}\right)\right. \\
&\left.+\beta^{2}\left(C e^{\beta \lambda z}+D e^{-\beta \lambda z}\right)\right] \lambda^{3} J_{0}(\lambda r) d \lambda \\
& F_{2}(r, z)= \int_{0}^{\infty}\left[(L+K)\left(A e^{\alpha \lambda z}+B e^{-\alpha \lambda z}\right)\right. \\
&\left.+(M+K)\left(C e^{\beta \lambda z}+D e^{-\beta \lambda z}\right)\right] \lambda^{2} J_{1}(\lambda r) d \lambda \\
& F_{3}(r, z)= \int_{0}^{\infty}\left(A e^{\alpha \lambda z}+B e^{-\alpha \lambda z}+C e^{\beta \lambda z}+D e^{-\beta \lambda z}\right) \lambda^{3} J_{0}(\lambda r) d \lambda
\end{aligned}
$$

and $J_{1}$ is the Bessel's function of first kind of order one.

\section{STATEMENT OF THE PROBLEM AND ITS SOLUTION}

The theory here given deals with a semi-infinite discrete, particulate granular layer of infinite extent in both the horizontal directions and the plane boundary of the upper surface is denoted by, $z=0$ plane. The $z$-axis is drawn with positive direction downward. Under an external load over a rigid circular area (a, being the radius); the stress and displacement fields in the granular layer are given by Eqs. (16) through (21), by taking $A=C=0$ so that the vertical normal stress $\left(\sigma_{z z}\right)$ and shear stress $\left(\tau_{r z}\right)$ tend to zero as $z \rightarrow \infty$ (Misra and Sen, 1975). In addition when it is prescribed that the surface is free from any shear stress; solution of Eq. (19) yields $B=-\beta^{2} D$. Hence for the surface $(z=0)$, Eq. (18) can be written as,

$$
\sigma_{z z}=-\int_{0}^{\infty}\left(1-\beta^{2}\right) D \lambda^{3} J_{0}(\lambda r) d \lambda
$$

Thus, the problem now reduces to determine the value of the function $(D)$ for different types of loading transmitted to the granular layer (Positive and/or negative boundary reactions, Fig. 1 ) through the contact surface with the rigid footing. The 


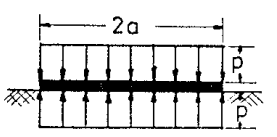

(a)

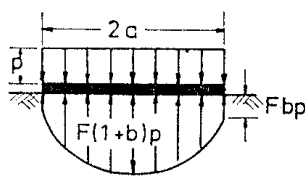

(b)

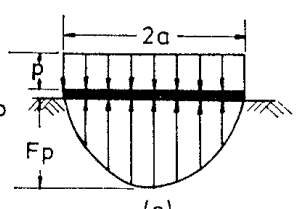

(c)

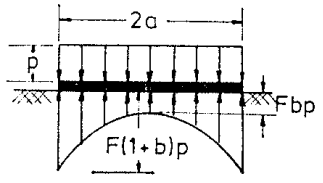

(d)

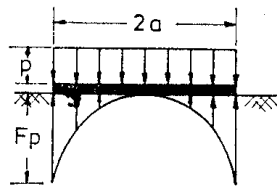

(e)

Fig. 1. Contact pressure on base of uniformly loaded circular plate

final solution of the function $(D)$ for various values of contact pressures and for different types of boundary conditions are available in writer's earlier works (1975-1976),

Once, $D$ is determined, based on the principle of reflection, the solution for lateral pressures against vertical retaining wall (the face taken to coincide with the $\mathrm{YZ}$ plane i. e, $r z, \theta=0$ plane in cylindrical polar co-ordinates) amounts to determining twice
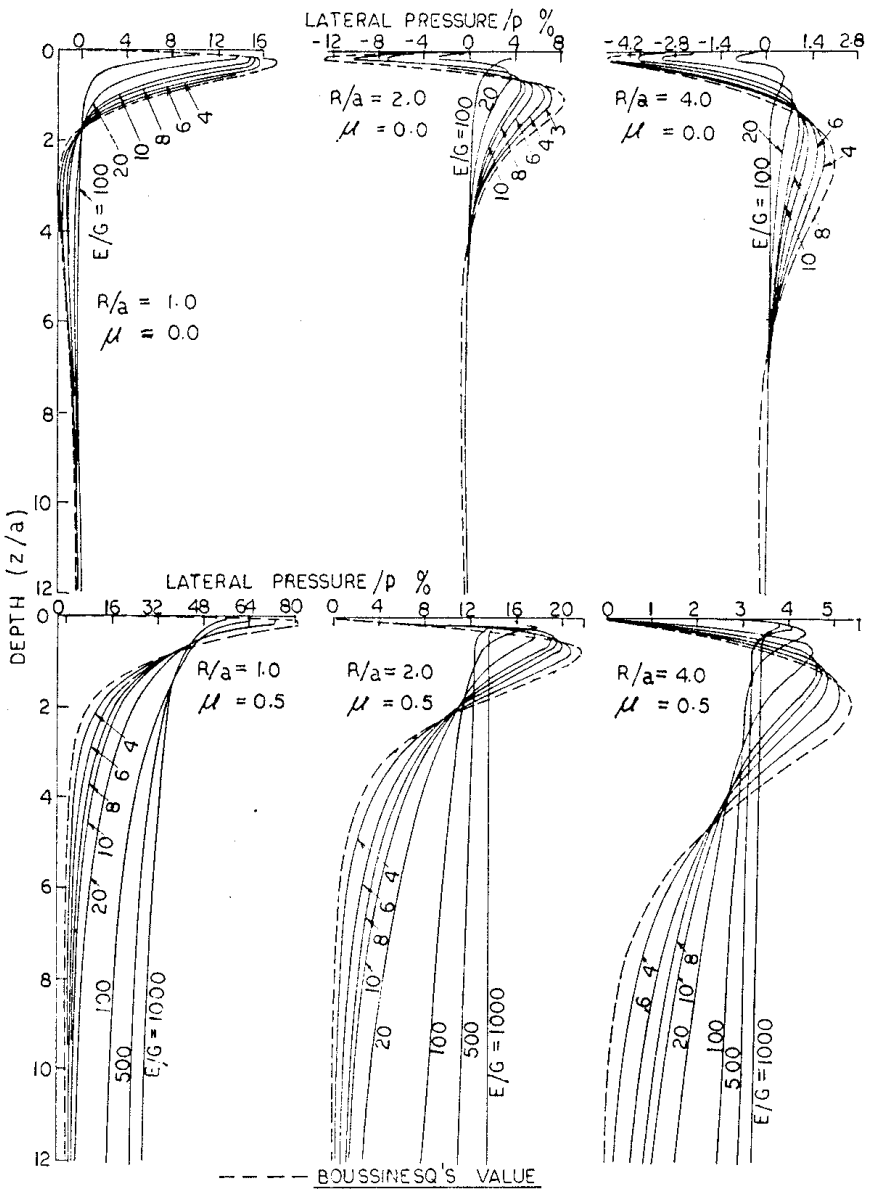

Fig. 2. Lateral pressure vs. depth (uniform contact pressure) 


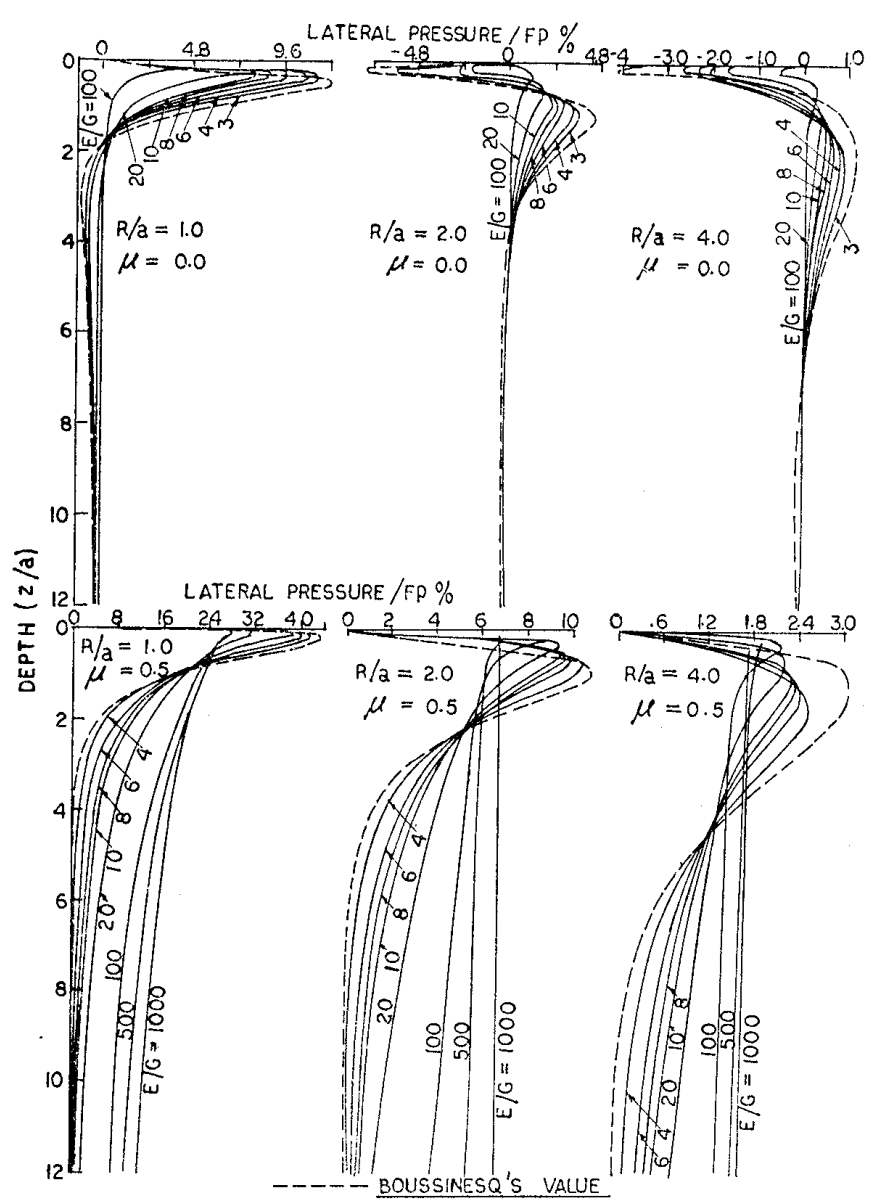

Fig. 3. Lateral pressure vs. depth (parabolic contact pressure)

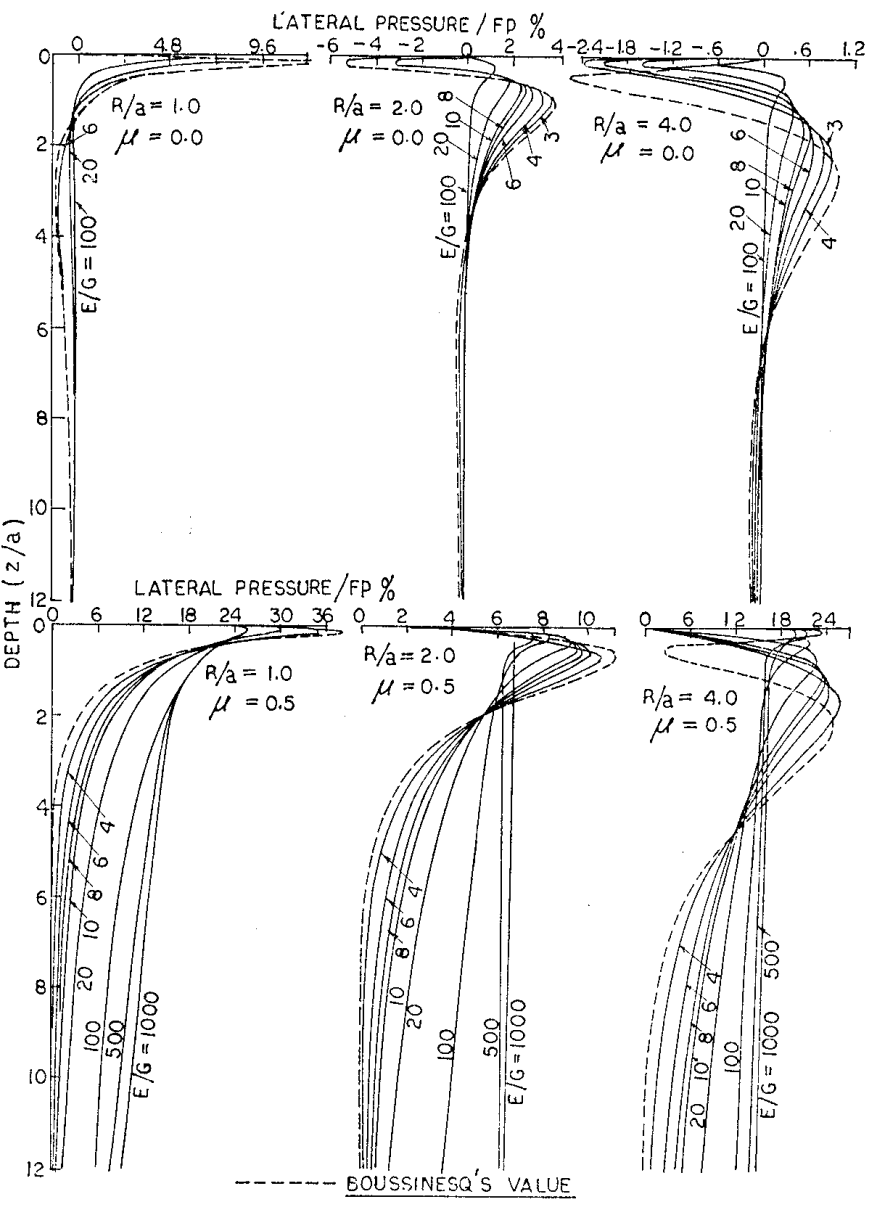

Fig. 4. Lateral pressure vs. depth (inverse parabolic contact pressure)

the value of $\left(\sigma_{r r}\right)$ across the plane $(r z, \theta=0)$ given by Eq. (16). The radial stress $\left(\sigma_{r r}\right)$ equations resulting from this material parameter $(E / G)$ concept for the various types of loading on the ground surface for different types of boundary conditions (at the bottom of the granular layer) are reproduced in the final form in Appendix-I for reference. Only the vertical retaining wall is treated in this paper and the wall friction is neglected.

\section{NUMERICAL RESULTS AND DISCUSSIONS}

In the absence of established values of the parameter $(E / G)$ for different material structures of the backfill, results have been presented for arbitrarily selected values of $E / G$, over a wide range, to understand its influence on the development of lateral pressures on walls retaining different types of backfill materials. Initially close intervals have been preferred as it is expected that most of the granular materials with which practical engineers deal with may have, $E / G$ values within 10 . Later on higher intervals have been selected for generalising, and understanding the versatility of, the material parameter concept. For the different values of Poisson's ratio the trend of the numerical results being similar and the quantitative values not much different the results for the two extreme values of $\nu$ are included here for a broader presentation; though it is a fact that, $\nu=0$ does not correspond to reality.

For the present analysis numerical evaluation of the integrals have been made by 


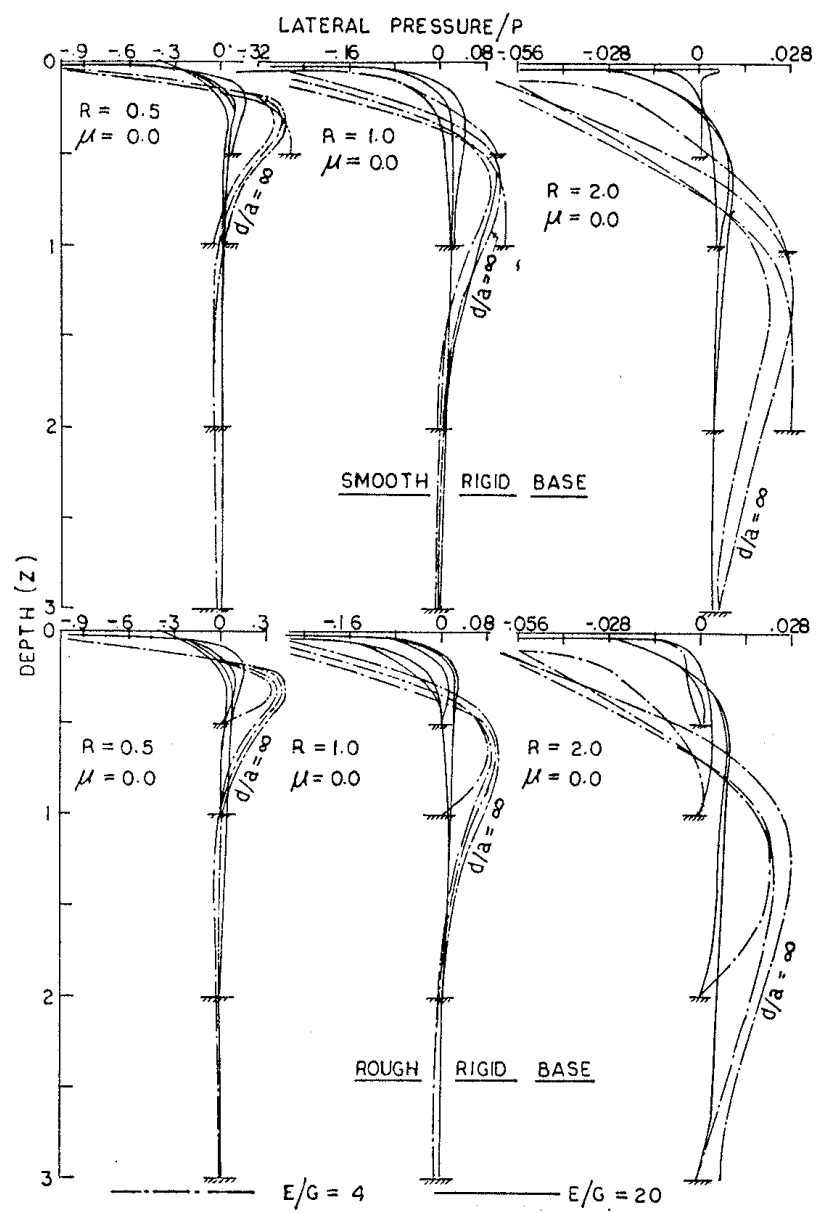

Fig. 5 (a). Lateral pressure vs. depthfinite layer (point load)

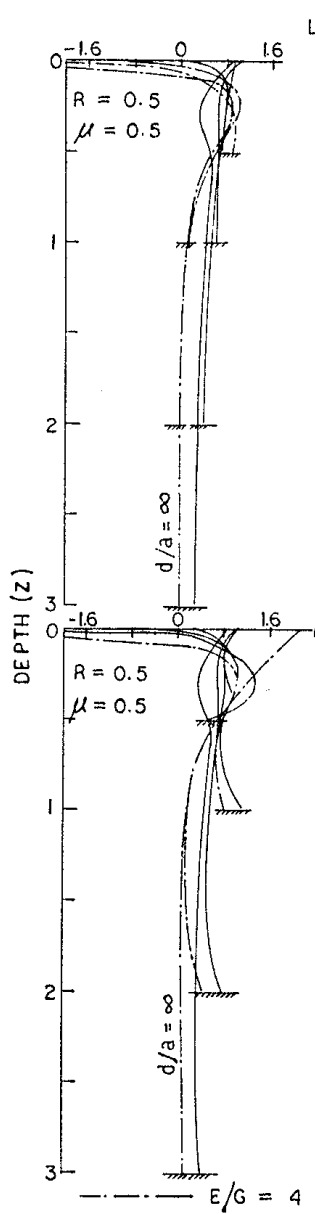

LATERAL PRESSURE/P

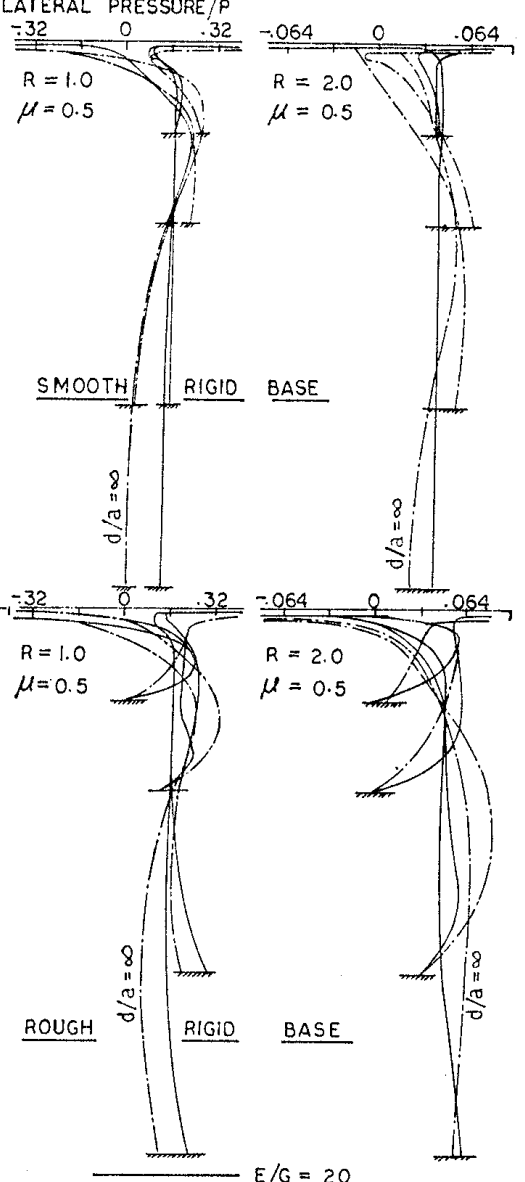

Fig. 5 (b). Lateral pressure vs. depthfinite layer (point load)

Simpson's method, as it has been found by the writer (1975 through 1977) that this simple method gives sufficiently accurate results for all practical purposes. Tension stress appears in the calculation of lateral earth pressures as presented in this paper only for certain conditions (Figs. 2 through 8 ). In general the tension stress appears for the materials exhibiting smaller values of the parameter $(E / G)$ and that too close to the surface of the backfill. Because of the confining effect, as per this concept it is expected that the backfill materials actually should exhibit very high values close to the surface and gradually decreasing with depth to 2 as given by the classical elastic relations, $E / G=2(1+\nu)$. It is hoped that by adopting a more practical variable parameter $(E / G)$ with respect to depth (a very high value at the surface gradually decreasing to that given by the classical relations), instead of a constant value of $(E / G)$ defined for the homogeneous backfill, a more realistic picture could be obtained.

The influence of $E / G$ ratio on the lateral pressure values, expressed as the percentage of the surcharge load, for the three cases; namely (I) the infinite depth, (II) the finite depth with rough and (III) with smooth interface; is presented in Figs. 2 through 8 for different types of contact pressures (Fig.1). The results have been presented for three different positions of the surcharge load measured as distance $(R)$ from the wall. For circular load the distance is measured upto the center of the die. The results given by the classical Boussinesq's theory have also been included 


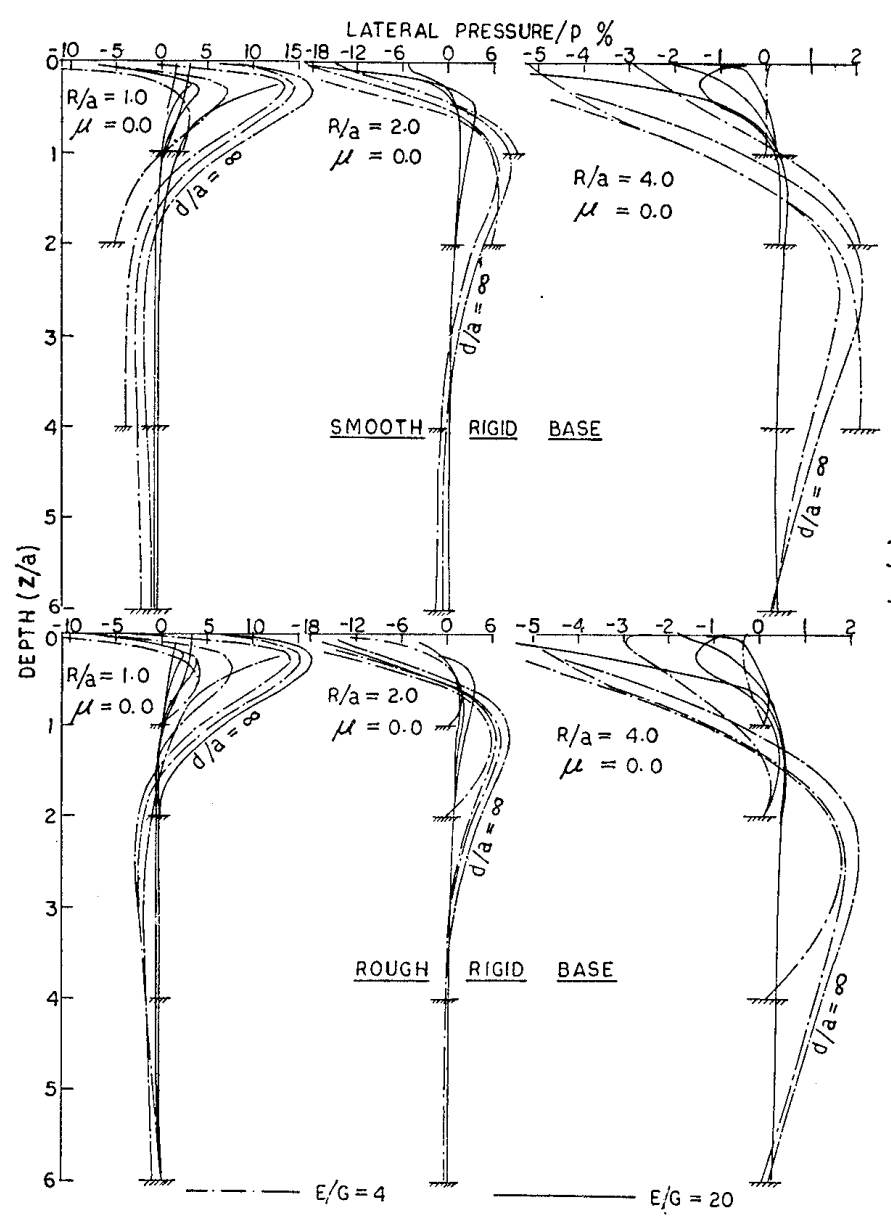

Fig. 6. (a) Lateral pressure vs. depthfinite layer (uniform contact pressure)

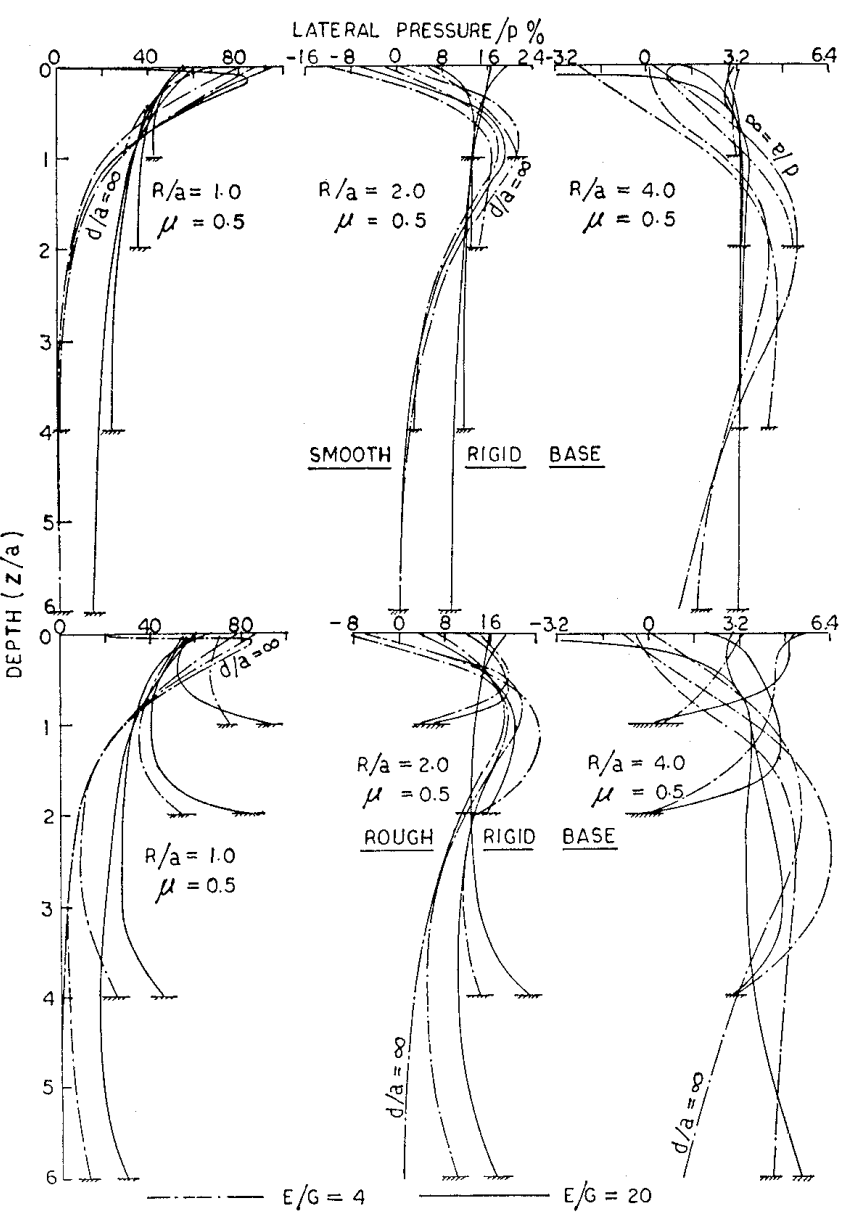

Fig. 6. (b) Lateral pressure vs. depthfinite layer(uniform contact pressure)

for the purpose of comparison.

It is felt that no useful purpose will be served by comparing these analytical values with the experimental results already published as every investigation had specific aim and purpose, and accordingly the variables reported in their works. Instead they are compared with the analytical results given by the classical Boussinesq's approach, in order to demonstrate the wider applicability of the present concept. Moreover, results considering a variable material parameter $(E / G)$ with depth will be a more realistic model once the concept is associated with the solution of these types of soil-structure interaction problems.

In the case of infinite depth (Figs. 2 through 4) it is observed that, for backfill materials having Poisson's ratio of 0.5 , the lateral pressure increases and tends to be equal all through the depth with the increase in the value of the material parameter $(E / G)$ irrespective of the position of the surcharge load from the wall face; a phenomenon characteristics of a liquid medium retained by a dam and supporting a load on its surface. However, the magnitude of the maximum value of the lateral pressure on the wall decreases with the increase in the distance of the load from the wall face, irrespective of the value of the parameter $E / G$. On the other hand for the Poisson's ratio equal to zero, though the tendency for equal lateral pressure all through the depth with the increase in the value of the parameter $E / G$ is observed, the magnitude of lateral pressure reduced with the increase in the value of the ratio $E / G$, and 


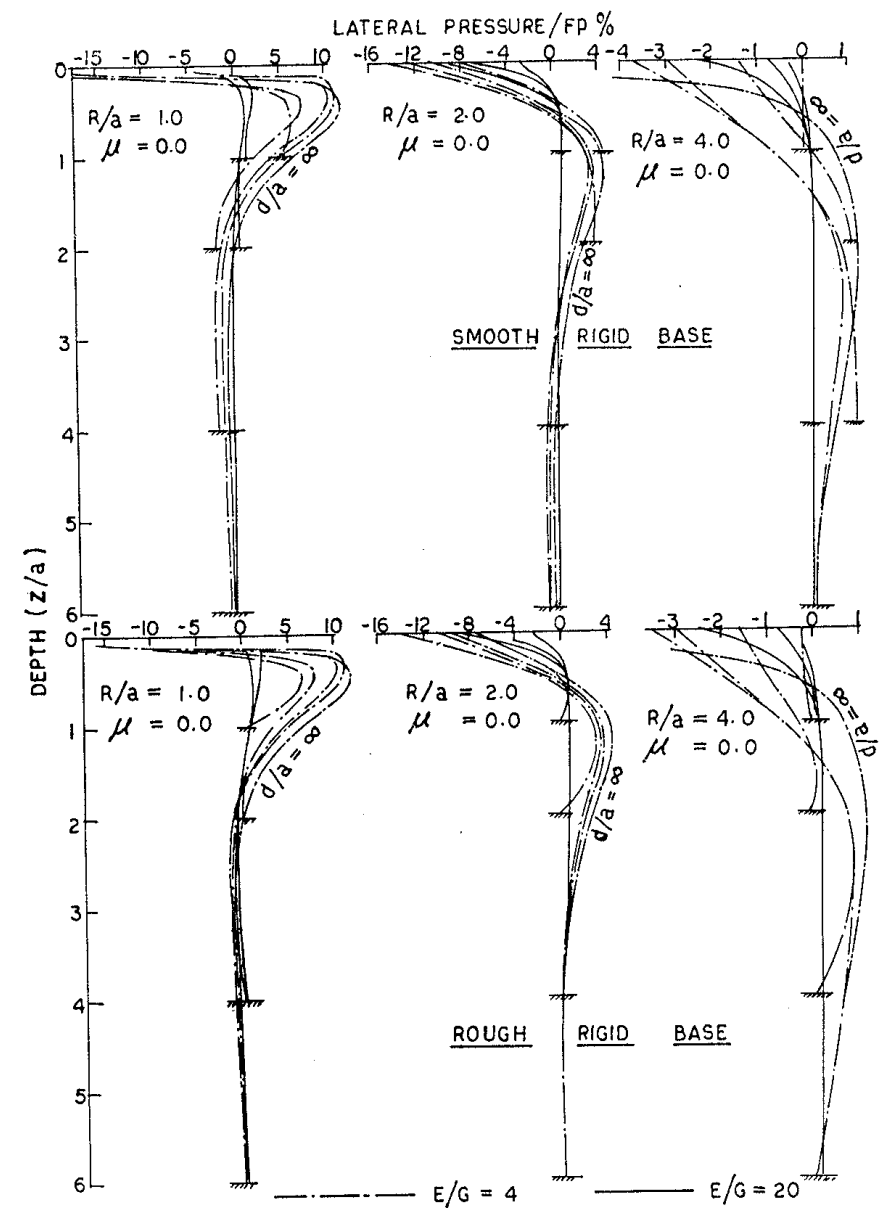

Fig. 7. (a) Lateral pressure vs. depthfinite layer(parabolic contact pressure)

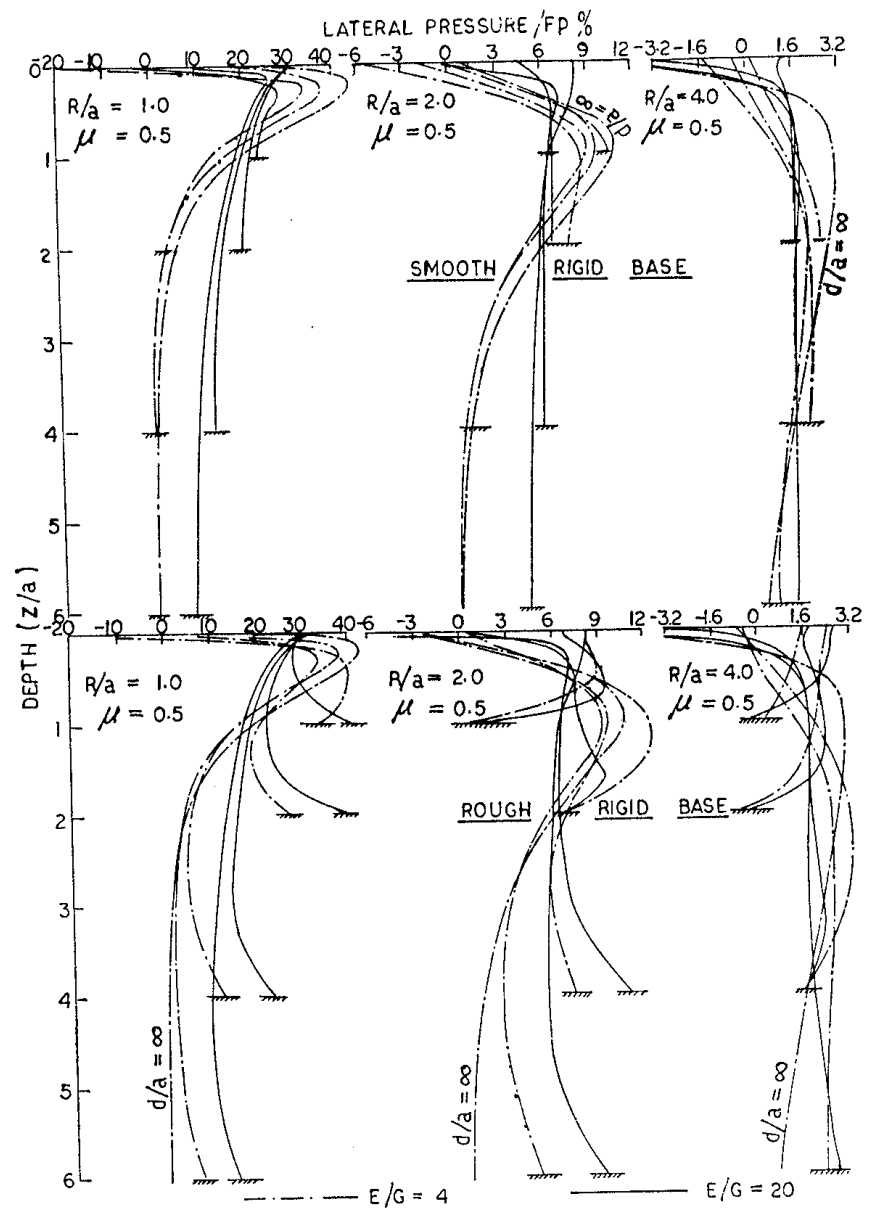

Fig. 7. (b) Lateral pressure vs. depthfinite layer (parabolic contact pressure)

reached a zero value for $E / G$ equal to 100 or more. In other respects the pattern of the curves of the present analysis compared well with the classical solution. Similar trend for the results with a Point load on the surface of the granular backfill has been reported by the writer (1977).

In the case of finite depth of the granular backfill (Figs. 5 through 8) results for only two values of the parameter, (E/G equal to 4 and 20), for four different thicknesses of the medium $(d)$ and for each thickness for the three different positions of the surcharge load away from the wall face $(R)$, have been presented along with the results for the infinite thick layer. The lateral pressure is observed to be higher for high values of $E / G$. It is noticed that the presence of a rough interface influenced considerably the development of lateral pressure for all values of Poisson's ratio; the influence being more pronounced for smaller thicknesses of the finite medium. The smooth interface as compared to the rough one influenced in a smaller degree.

\section{SUMMARY AND CONCLUSIONS}

An attempt has been made to elucidiate the physical and mechanical properties of the materials responsible for the lateral stress transmittal. It gives a partial explanation of the divergences between the results of the existing theories and reality. 


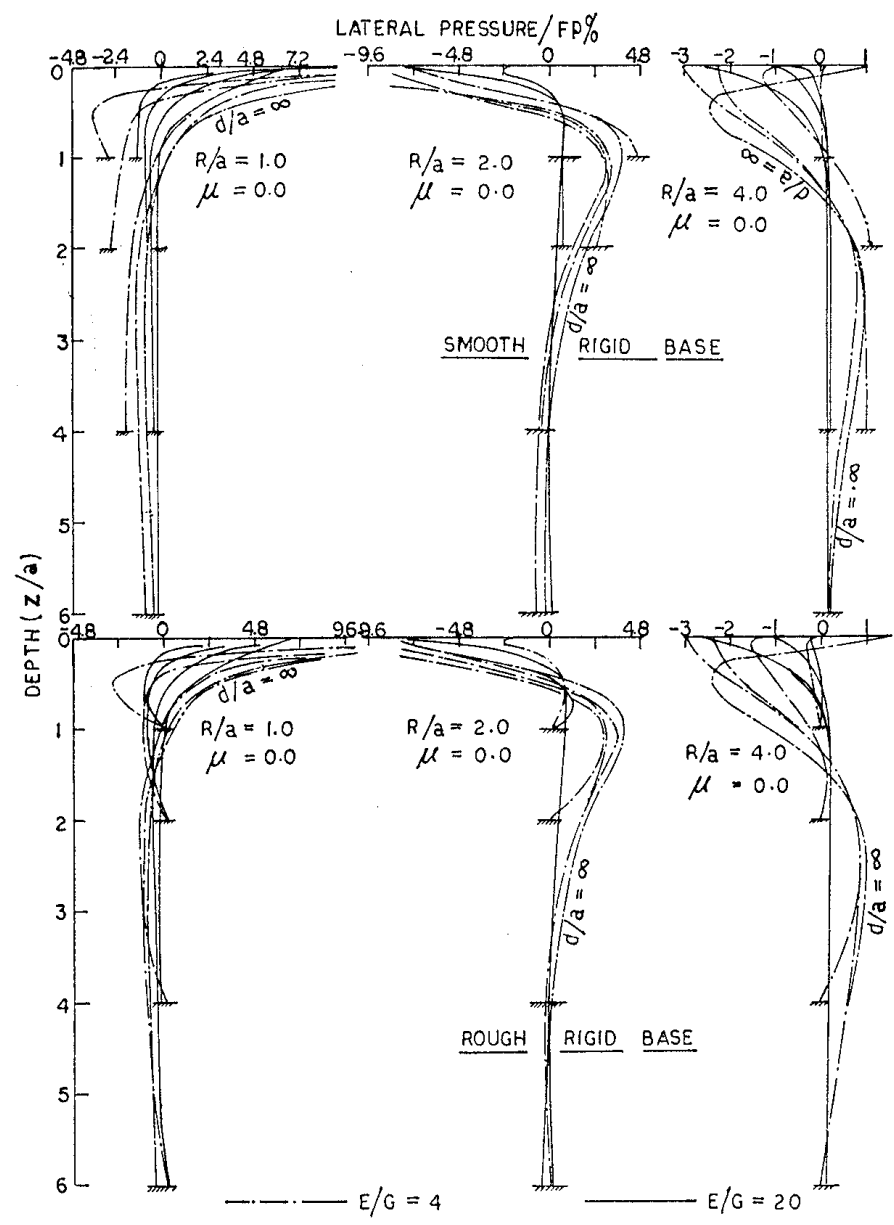

Fig. 8. (a) Lateral pressure vs. depth-finite layer (inverse parabolic contact pressure)

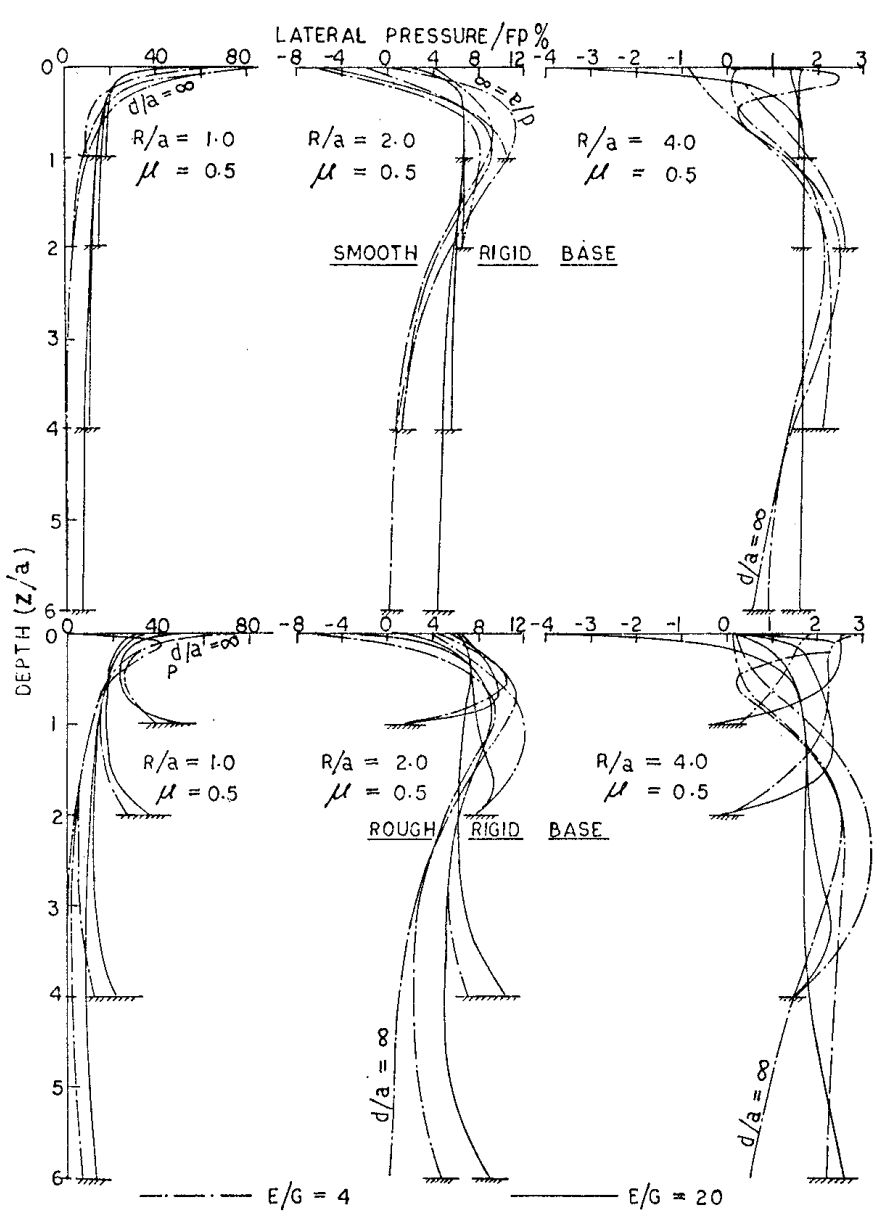

Fig. 8. (b) Lateral pressure vs. depth-finite layer (inverse parabolic contact pressure)

The inability of certain materials of the nature; such as-liquids, saturated soils, powdery substances like mineral dusts, fertilisers, particulate materials like paddy grains, coal and ores etc.; to transmit the vertical force in the horizontal direction is clearly indicated by the present model. It is observed that the softer/looser the backfill medium (represented by high $E / G$ values), the poorer is lateral stress transmission resulting in a overall decrease of the lateral pressure on the retaining structures.

Information available from actual structures on the subject at present is insufficient to enable one to state that any particular method of calculating lateral stress distributions due to surcharge load is to be preferred over the others. More experiments with different backfill materials and at different conditions of state are required in greater detail to study the effect of the various physical parameters on the lateral stress transmission in loose particulate medium and to find more realistic expressions for predicting the stress pattern in different types of backfill materials ranging from solid like at one end to liquid like at the other end.

As an extension of the mathematical techniques developed herein, one may consider the problem of a variable material parameter $(E / G)$ in some practical form, for which no solutions are available. The variable material parameter $(E / G)$ has specific use in these type of problems since the load on the backfill surface can be located outside the plastic zone of the backfill for certain values of $(R / a)$. As in this concept the value of the parameter $(E / G)$ also takes into account the state of the material, the 
corresponding value of the parameter $(E / G)$ is to be used for the elastic and plastic zones, determined specifically for that state of the material. It is expected that the techniques developed herein would prove successful in these case too, although the numerical calculations may be laborious.

\section{APPENDIX-I}

The development of the equations; given in this Appendix, which are the logical solutions of the Eqs. (16) and (25), being similar to that reported in writer's $(1975,1976,1977)$ published works cited under references, wherever possible reference is given to these works instead of giving elaborate simplifications.

Case-I. Infinite Depth of the Granular Backfill (Misra and Sen, 1975)

For this problem the radial stress $\left(\sigma_{r r}\right)$ equations due to different types of surcharge load on the surface of the backfill (Fig.1(a) 1(e)) are as follows:

Point load (Misra, 1977)

$$
\begin{aligned}
\sigma_{r r}= & \frac{P}{2 \pi(\alpha-\beta)}\left[z^{-2}\left[\alpha^{2}\left[\alpha^{2}+(r / z)^{2}\right]^{-3 / 2}-\beta^{2}\left[\beta^{2}+(r / z)^{2}\right]^{-3 / 2}\right]\right. \\
& \left.-r^{-2}\left[(L+K)\left[\beta-\left[\alpha^{2}+(r / z)^{2}\right]^{-1 / 2}\right]-(M+K)\left[\alpha-\left[\beta^{2}+(r / z)^{2}\right]^{-1 / 2}\right]\right]\right]
\end{aligned}
$$

Uniform contact pressure (Misra and Sen, 1975)

$$
\begin{aligned}
\sigma_{r r} & =\frac{a p}{\alpha-\beta} \int_{0}^{\infty} F_{4}(r, z) J_{1}(\lambda a) d \lambda \\
\text { where, } F_{4}(r, z)=[ & {\left[\alpha\left(e^{-\alpha \lambda z}-\beta e^{-\beta \lambda z}\right)\right] J_{0}(\lambda r)-\left[\beta(L+K) e^{-\alpha \lambda z}\right.} \\
& \left.\left.-\alpha(M+K) e^{-\beta \lambda z}\right] \frac{1}{\lambda r} J_{1}(\lambda r)\right]
\end{aligned}
$$

Parabolic contact pressure (Negative boundary reactions)

For the parabolic contact pressure (Fig. 1 (c)) having zero pressure at the periphery of the footing and maximum at the centre (i.e. $b=0$ ) the solution takes the form (Misra and Sen, 1975);

$$
\sigma_{r r}=\frac{2 F p}{\alpha-\beta} \int_{0}^{\infty} F_{4}(r, z) \frac{1}{\lambda} J_{2}(\lambda a) d \lambda
$$

in which, $F$ and $b$ are multiplying factors and $J_{2}$ is the Bessel's function of the first kind of order two.

Inverse parabolic contact pressure (Positive boundary reactions)

For the inverse parabolic contact pressure (Fig. 1(e)) having zero pressure at the centre of the footing and maximum at the periphery (i.e. $b=0$ ) the solution is given by (Misra and Sen, 1975);

$$
\sigma_{r r}=\frac{F p}{\alpha-\beta} \int_{0}^{\infty} F_{4}(r, z)\left[a J_{1}(\lambda a)-\frac{2}{\lambda} J_{2}(\lambda a)\right] d \lambda
$$

Case-II. Finite Depth of the Granular Backfill Underlain by a Rough Rigid Base (Misra and Sen, 1976)

For this problem the equations for the lateral pressures on vertical retaining wall due to different types of surcharge load on the surface of the backfill are as follows: 
Point load

$$
\sigma_{r r}=\frac{P}{4 \pi} \int_{0}^{\infty} F_{5}(r, z) \lambda d \lambda
$$

Uniform contact pressure (Fig. 1(a))

$$
\sigma_{r r}=\frac{a p}{2} \int_{0}^{\infty} F_{5}(r, z) J_{1}(\lambda a) d \lambda
$$

Parabolic type contact pressure (Fig.1(c))

$$
\sigma_{r r}=F p \int_{0}^{\infty} F_{5}(r, z) \frac{1}{\lambda} J_{2}(\lambda a) d \lambda
$$

Inverse parabolic type contact pressure (Fig. 1(e))

$$
\begin{gathered}
\sigma_{r r}=\frac{F p}{2} \int_{0}^{\infty} F_{5}(r, z)\left[a J_{1}(\lambda a)-\frac{2}{\lambda} J_{2}(\lambda a)\right] d \lambda \\
\text { where, } F_{5}(r, z)=\left[\frac{1}{\lambda r} g_{6}(\lambda) J_{1}(\lambda r)-g_{7}(\lambda) J_{0}(\lambda r)\right]
\end{gathered}
$$

in which, $g_{6}(\lambda)$ and $g_{7}(\lambda)$ are trigonometric functions, which are reported in full details in writer's work under reference (1976).

Case-III. Finite Depth of the Granular Backfill Underlain by a Smooth Rigid Base (Misra and Sen, 1976)

For this problem the equations for the lateral pressures are;

Point load

$$
\sigma_{r r}=\frac{P}{2 \pi} \int_{0}^{\infty} F_{6}(r, z) \lambda d \lambda
$$

Uniform contact pressure (Fig.1(a))

$$
\sigma_{r r}=a p \int_{0}^{\infty} F_{6}(r, z) J_{1}(\lambda a) d \lambda
$$

Parabolic type contact pressure (Fig. 1(c))

$$
\sigma_{r r}=2 F p \int_{0}^{\infty} F_{6}(r, z) \frac{1}{\lambda} J_{2}(\lambda a) d \lambda
$$

Inverse parabolic type contact pressure (Fig. 1(e))

$$
\begin{gathered}
\sigma_{r r}=F p \int_{0}^{\infty} F_{6}(r, z)\left[a J_{1}(\lambda a)-\frac{2}{\lambda} J_{2}(\lambda a)\right] d \lambda \\
\text { where, } F_{6}(r, z)=\left[\frac{1}{\lambda r} f_{1}(\lambda) J_{1}(\lambda r)+f_{2}(\lambda) J_{0}(\lambda r)\right]
\end{gathered}
$$

in wihch, $f_{1}(\lambda)$ and $f_{2}(\lambda)$ are trigonometric functions, which are available with full details in writer's work under reference (1976).

The value of the coefficient $F$, shown in Eqs. (29), (30), (33), (34), (38), (39) and in Figs. 3, 4, 7 and 8, depends on the type of contact pressure developed (Figs. 1 (b) through 1(e)). From the geometry of the contact pressure developed the value of the coefficient $F$ is obtained.

\section{NOTATION}

$A, B, C, D=$ arithmatic functions 
$E, G, \nu=$ elastic constants of the granular medium

$F, b=$ multiplying factors

$F_{1}, \cdots, F_{6}=$ mathematical functions

$J_{0}, J_{1}, J_{2}=$ Bessel functions of first kind of order zero, one and two respectively

$$
\begin{aligned}
K & =\frac{E}{1+\nu}\left(\frac{1}{G}-\frac{1+\nu}{E}\right)>1 \\
K^{\prime} & =(1-K) /(1-\nu) \\
L & =(K-1) /\left(\alpha^{2}-1\right) \\
M & =-\alpha^{2} L
\end{aligned}
$$

$P, p=$ surcharge loads-concentrated and uniform respectively

$R=$ distance of the surcharge load from the face of the retaining wall

$a=$ radius of the circular footing

$\alpha^{2}, \beta^{2}=$ roots of the equation, $x^{2}+\left(K^{\prime}-2\right) x+1=0$

$d=$ thickness of the finite granular layer

$\nabla^{2}=$ Laplacian operator

$e=$ exponential function

$f_{1}(\lambda), f_{2}(\lambda)=$ trigonometric functions

$g_{6}(\lambda), g_{7}(\lambda)=$ trigonometric functions

$\lambda=$ trigonometric variable

$\phi, \psi=$ stress functions

$r, \theta, z=$ radial angular and depth co-ordinates respectively

$\sigma_{r r}, \sigma_{\theta \theta}, \sigma_{z z}=$ normal stresses

$\tau_{r z}=$ shear stress

$u, w=$ radial and axial components of displacements

\section{REFERENCES}

1) Barber, E.S. (1956): "Discussion, Lateral pressures on retaining walls due to backfill surface loads," HRB, Bull, 141, pp. 15-18.

2) Boussinesq, J. (1885): “Application des Potentiels and L'Etude de I'E' euilibre et du Mouvement des Solides E'lastques," Gauthier-Villars.

3) Hansen, J.B. (1953): Earth Pressure Calculation, The Danish Technical Press, Copenhagen.

4) Ichihara, M. and Matsuzawa, H. (1970): "Correlations between properties of earth pressure on tilting wall and the shearing characteristics of dry backfill sand," Trans. of JSCE, Vol.2, Pt. 1 , pp. 91-95.

5) Massarsch, K. R. and Broms, B. B. (1976) : "Lateral earth pressure at rest in soft clay," Proc., ASCE, Vol.102, No. GT 10, pp. 1041-1047.

6) Matsuo, M., Kenmochi, S. and Yagi, H. (1978): "Experimental study on earth pressure of retaining wall by field tests," Soils and Foundations, Vol.18, No. 3, pp. 27-41.

7) Mindlin, R.D. (1936) : "Discussion, Pressure distribution on retaining walls," Proc., 1 st. ICSMFE, Harvard University, Vol. III, pp. 155.

8) Misra, B. (1977) : "Lateral pressure due to surface load on granular backfill," Proc., Int. Symp. on Soil Structure Interaction, Univ, of Roorkee, India, Vol.1, pp.73-77.

9) Misra, B. and Sen, B.R. (1975): "Stresses and displacements in granular materials due to surface load," Int. J. Engng. Sci., Vol.13, No.7-8, pp.743-761.

10) Misra, B. and Sen, B. R. (1976): "Surface loads on granular material underlain by a rough rigid base," Int. J. Engng. Sci., Vol.14, No. 3, pp. 319-334.

11) Misra, B. and Sen, B.R. (1976): "Surface loads on granular material underlain by a smooth rigid base," Letters in Applied and Engng. Sci., an Int. J., Vol. 4, No. 4, pp. 297-311.

12) Ochiai, H. (1977) : "Expression of the coefficient of earth pressure at rest of sand and a calculation method using $N$-value," Soils and Foundations, Vol.17, No.3, pp. 93-101.

13) Richard, O.D. and Linger, D.A. (1965): “An experimental study of lateral pressures on 
abutment retaining walls," HRR, No. 74 , pp. 19-43.

14) Spangler, M. G. (1938) : "Lateral pressures on retaining walls caused by superimposed loads," Proc., HRB, Vol.18, Pt.2, pp.9-181.

15) Spangler, M. G. and Mickle, J.L. (1956): "Lateral pressures on retaining walls due to backfill surface loads," HRB, Bull, 141, pp.1-15.

16) Terzaghi, K. (1943): Theoretical Soil Mechanics, John Wiley Inc.

17) Timoshenko, S. (1934): Theory of Elasticity, McGraw-Hill Book Co., New York.

18) Tschebotarioff, G.P. (1957) : Soil Mechanics, Foundations and Earth Structures, McGraw-Hill Book Co., New York.

19) Weiskopf, W. H. (1945) : "Stresses in soils under a foundation," J. Franklin Inst. Vol.239, No. 6, pp. 445-465.

(Received March 10, 1979) 